\section{(6) OPEN ACCESS}

\title{
Characterising the progress in HIVIAIDS research in the Middle East and North Africa
}

\author{
Hanan F Saba, ${ }^{1}$ Silva P Kouyoumjian, ${ }^{1}$ Ghina R Mumtaz, ${ }^{1}$ Laith J Abu-Raddad ${ }^{1,2,3}$
}

\section{Arabic Abstract translation \\ ${ }^{1}$ Infectious Disease \\ Epidemiology Group, Weill Cornell Medical College - Qatar, Qatar Foundation, Education city, Doha, Qatar ${ }^{2}$ Department of Public Health, Weill Cornell Medical College, Cornell University, New York, USA \\ ${ }^{3}$ Vaccine and Infectious Disease Division, Fred Hutchinson Cancer Research Center, Seattle, Washington, USA}

\section{Correspondence to} Dr Laith J Abu-Raddad, Infectious Disease Epidemiology Group, Weill Cornell Medical College Qatar, Qatar Foundation, Education City,

P.O. Box 24144, Doha Qatar: lja2002@qatar-med.cornell.edu

Received 25 October 2012 Revised 27 February 2013 Accepted 18 March 2013 Published Online First 17 April 2013

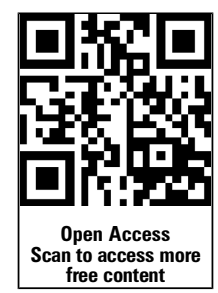

To cite: F Saba $\mathrm{H}, \mathrm{P}$ Kouyoumjian $S, R$

Mumtaz G, et al. Sex

Transm Infect 2013;89:

iii5-iii9.
ABSTRACT

Objectives The Middle East and North Africa (MENA) region is perceived to have limited HIV data. The objective of this study was to quantitatively characterise the progress in HIV research in this region since the discovery of the epidemic.

Methods Four indices were defined and implemented to measure the progress of HIV research using the PubMed, Embase, MENA HIVIAIDS Epidemiology Synthesis Project and US Census Bureau HIVIAIDS Surveillance databases. The four indices provide complementary measures to characterise different aspects of the progress of HIV research.

Results A total of 2118, 2352, 683 and 4889 records were identified through the PubMed, the Embase, the Synthesis Project and the HIV Prevalence indices, respectively. The proportion of the total global HIV records that relate to MENA is $1.2 \%$. Overall, the indices show steady progress in the number of new records every year, with an accelerated pace in the last few years. The rate of progress in MENA was also higher than the rate of progress in HIV records globally. There is no evidence so far of stabilisation or a peak in the number of new records year by year. About half of the records were produced after the year 2005. The number of records shows large heterogeneity across countries. Conclusions MENA has witnessed a rapid growth in HIV research over the last decade. However, there are still large gaps in HIV scientific evidence in the region, and the progress is far from being uniform across countries. Ongoing and future research needs to be geared towards academic standard and production of scientific publications.

\section{INTRODUCTION}

The Middle East and North Africa (MENA) region is viewed as the anomaly in the HIV/AIDS world map and 'a real hole in terms of HIV/AIDS epidemiological data'. ${ }^{1}$ The region is also perceived to have failed to address the HIV public-health challenge, and in denial of the reality of the HIV epidemic in this part of the world. ${ }^{2}{ }^{3}$ The aim of this article is to document and quantitatively characterise the growth in HIV research in MENA over the last three decades since the discovery of the epidemic.

\section{METHODOLOGY}

We defined and implemented four indices for measuring the progress of HIV research in MENA. These correspond to the number of publications or data retrieved through a search of four different sources of data. The indices are as follows:
- PubMed Index: The number of records identified through a search of studies on HIV in MENA in PubMed (Medline) database using a strategy with both free text and $\mathrm{MeSH}$ headings, and with no language or year restrictions. $\mathrm{MeSH}$ headings were exploded to cover all subheadings. The cut-off date for our search was 9 October 2012. The search criteria can be found in box 1 .

- Embase Index: The number of records identified through a comprehensive search of studies on HIV in MENA in Embase database using a strategy with both free text and Emtree terms, and with no language or year restrictions. Emtree terms were exploded to cover all subheadings. The cut-off date for our search was 9 October 2012. The search criteria can be found in box 1 .

- MENA HIV/AIDS Epidemiology Synthesis Project Index: The number of records that are not part of either the PubMed or the Embase databases, but are part of the MENA HIV/AIDS Epidemiology Synthesis Project EndNote database. $^{4}$ These records include predominantly country-level or international organisation reports, all of which have not been published in the scientific literature. These documents were obtained through our network formed by working with the countries and different national and international organisations in this region. These documents were compiled individually based on their actual relevance to HIV in MENA.

- HIV Prevalence Index: The number of HIV point-prevalence surveys as extracted from the US Census Bureau HIV/AIDS Surveillance database, ${ }^{5}$ a comprehensive compilation of global HIV prevalence measures irrespective of collection methodology. The cut-off date for inclusion was 9 October 2012. The numbers quoted for Sudan includes both Sudan and the newly formed Republic of South Sudan.

The four indices capture different aspects of the progress of HIV research in MENA, and each one complements or corrects for potential biases in the other indices. While the PubMed and Embase indices characterise the progress in terms of published HIV research in the scientific literature, the Synthesis Project Index characterises the progress in the unpublished grey literature. This is essential as a large volume of HIV studies in MENA have never been published in scientific journals due to multiple obstacles. ${ }^{4}{ }^{6}$ Although both the PubMed 
Box 1 Details of the search criteria used to search

PubMed and Embase databases for relevant articles

PubMed Index Search Criteria

1. HIV in Middle East and North Africa (MENA) search

('HIV'[Mesh] OR 'HIV Seropositivity'[Mesh] OR 'HIV

Antibodies'[Mesh] OR 'HIV Infections'[Mesh] OR 'HIV

Seroprevalence'[Mesh] OR HIV [Text] or 'Human

immunodeficiency virus'[Text]) AND ('Middle East'[Mesh] OR

'Islam'[Mesh] OR 'Arabs'[Mesh] OR 'Arab World'[Mesh] OR

'Africa, Northern'[Mesh] OR 'Sudan'[Mesh] OR 'Somalia'[Mesh]

OR 'Dijibouti'[Mesh] OR 'Pakistan'[Mesh] OR 'Middle East'[Text] OR

'Middle-East'[Text] OR 'North Africa'[Text] OR 'North-Africa'[Text] OR

'EMRO'[Text] OR 'Eastern Mediterranean'[Text] OR 'Arab'[Text] OR

'Arabs'[Text] OR 'Arab World'[Text] OR 'Islam'[Text] OR

'Afghanistan'[Text] OR 'Algeria'[Text] OR 'Bahrain'[Text] OR

'Djibouti'[Text] OR 'Egypt'[Text] OR 'Jordan'[Text] OR 'Kuwait'[Text]

OR 'Lebanon'[Text] OR 'Libya'[Text] OR 'Iran' [Text] OR 'Iraq'[Text]

OR 'Morocco'[Text] OR 'Oman'[Text] OR 'Pakistan'[Text] OR

'Qatar'[Text] OR 'Saudi Arabia'[Text] OR 'Somalia'[Text] OR

'Sudan'[Text] OR 'Syria'[Text] OR 'Tunisia'[Text] OR 'United Arab

Emirates'[Text] OR 'Dubai'[Text] OR 'Abu Dhabi'[Text] OR

'Abu-Dhabi'[Text] OR 'Sharjah'[Text] OR 'West Bank'[Text] OR

'Ghaza'[Text] OR 'Palestine'[Text] OR'Yemen'[Text]).

2. Global HIV search

'HIV'[Mesh] OR 'HIV Seropositivity'[Mesh] OR 'HIV

Antibodies'[Mesh] OR 'HIV Infections'[Mesh] OR 'HIV

Seroprevalence'[Mesh] OR HIV [Text] or 'Human

immunodeficiency virus'[Text]

\section{Embase Index Search Criteria \\ 1. HIV in MENA search}

(exp Human immunodeficiency virus/ or HIV.mp. or Human immunodeficiency virus.mp.) AND (exp Middle East/ or exp North Africa/ or exp Arab/ or exp Afghanistan/ or exp Djibouti/ or exp Pakistan/ or exp Somalia/ or exp Sudan/ or Middle East.mp. or North Africa.mp. or EMRO.mp. or Eastern Mediterranean.mp. or Arab.mp. or Arabs.mp. or Arab World.mp. or Islam.mp. or Afghanistan.mp. or Algeria.mp. or Bahrain.mp. or Djibouti.mp. or Egypt.mp. or Jordan.mp. or Kuwait.mp. or Lebanon.mp. or Libya. $\mathrm{mp}$. or Iran.mp. or Iraq.mp. or Morocco.mp. or Oman.mp. or Pakistan.mp. or Qatar.mp. or Saudi Arabia.mp. or Somalia.mp. or Sudan.mp. or Syria.mp. or Tunisia.mp. or United Arab Emirates. mp. or Dubai.mp. or Abu Dhabi.mp. or Sharjah.mp. or West Bank. mp. or Ghaza.mp. or Palestine.mp. or Yemen.mp).

\section{Global HIV search}

exp Human immunodeficiency virus/ or HIV.mp. or Human immunodeficiency virus.mp.

and Embase indices focus on the published literature, there are differences in the sources of literature that are indexed in each of these databases. Embase has also the advantage of including additional sources of data that appeared only in conference abstracts. Similarly, while the first three indices cover a wide range of HIV-related studies including biological, behavioural and other contextual studies, the HIV Prevalence Index strictly measures the progress of HIV research in producing objective biomarkers of the level of HIV infection in various population groups.

Lastly, we compared the contribution of MENA to the total global HIV research output using the PubMed Index to quantify the magnitude and trend of MENA contribution relative to the global contribution. The search criteria for the global HIV research search can be found in box 1 .

\section{RESULTS}

We identified a total number of 2118 records through the PubMed Index, 2352 records through the Embase Index, 683 records through the Synthesis Project Index and 4889 records through the HIV Prevalence Index. The year-by-year distribution of each of these indices can be seen in figure 1. Overall, the indices collectively show steady increase in the number of new records every year, with an accelerated growth during the last few years. While still small, the fraction of MENA HIV records in PubMed, relative to global HIV records, has steadily increased from insignificant levels in earlier years to $1.2 \%$ in 2011 (figure 2A). More than half of the MENA records were produced only in the last few years, around 2005 and subsequent years. Records on HIV in MENA started to appear in the mid-1980s, but at a slow rate. There is no evidence so far of stabilisation or a peak in the number of new records year by year, suggesting that HIV research continues to be in an expansion phase.

Figure $2 \mathrm{~B}$ shows the distribution of studies by country through the HIV Prevalence Index. The number of records per country shows large heterogeneity with few countries producing hundreds of prevalence measures, while others having few available measures. Morocco, for example, has the highest number of records at 1089 , while only one prevalence measure was found for the United Arab Emirates.

\section{DISCUSSION}

The contribution of MENA to the global HIV literature is still meagre at just over 1\%, though MENA's population comprises nearly $10 \%$ of the world's population. Nevertheless, the results of our study indicate that MENA has witnessed a rapid growth in HIV research over the last decade. The results were consistent on this conclusion regardless of the index used to measure the progress. The pace of growth continues and at a faster rate compared to the global literature (figure 2A). The widely held perceptions of limited HIV data in MENA, if not inaccurate, exaggerate the limitations of our epidemic knowledge in this region. Though there are still large gaps in HIV evidence, these should not prevent us from appreciating what has been achieved in just a few years over the last decade.

This advancement in the quantity of studies masks a remarkable progress in the quality of studies, which has greatly improved over the last few years. Studies in earlier years tended to have methodological limitations, were mostly descriptive or qualitative in nature and were conducted on convenience samples that are not necessarily representative, such as facility-based or venue-based samples. ${ }^{46-8}$ However, the region has witnessed recently a clear shift in the nature of evidence with multiple studies being conducted with state-of-the-art research methodologies (further discussion of study quality can be found in ${ }^{4}{ }^{6-8}$ ). An example of quality studies are the integrated bio-behavioural surveillance surveys using innovative sampling methodologies for hard to reach populations such as respondent-driven sampling. Some countries such as Afghanistan, Egypt, Iran, Pakistan and Tunisia have now several rounds of surveillance among high-risk populations providing longitudinal data to track the evolution of the epidemic. ${ }^{9}$ Other countries have conducted their first round of surveillance; and in most of these, subsequent rounds are either planned or being implemented. ${ }^{7-9}$ All in all, the last decade in MENA can be described as a 'brave new world' in HIV research, after years in which the mere recognition that 

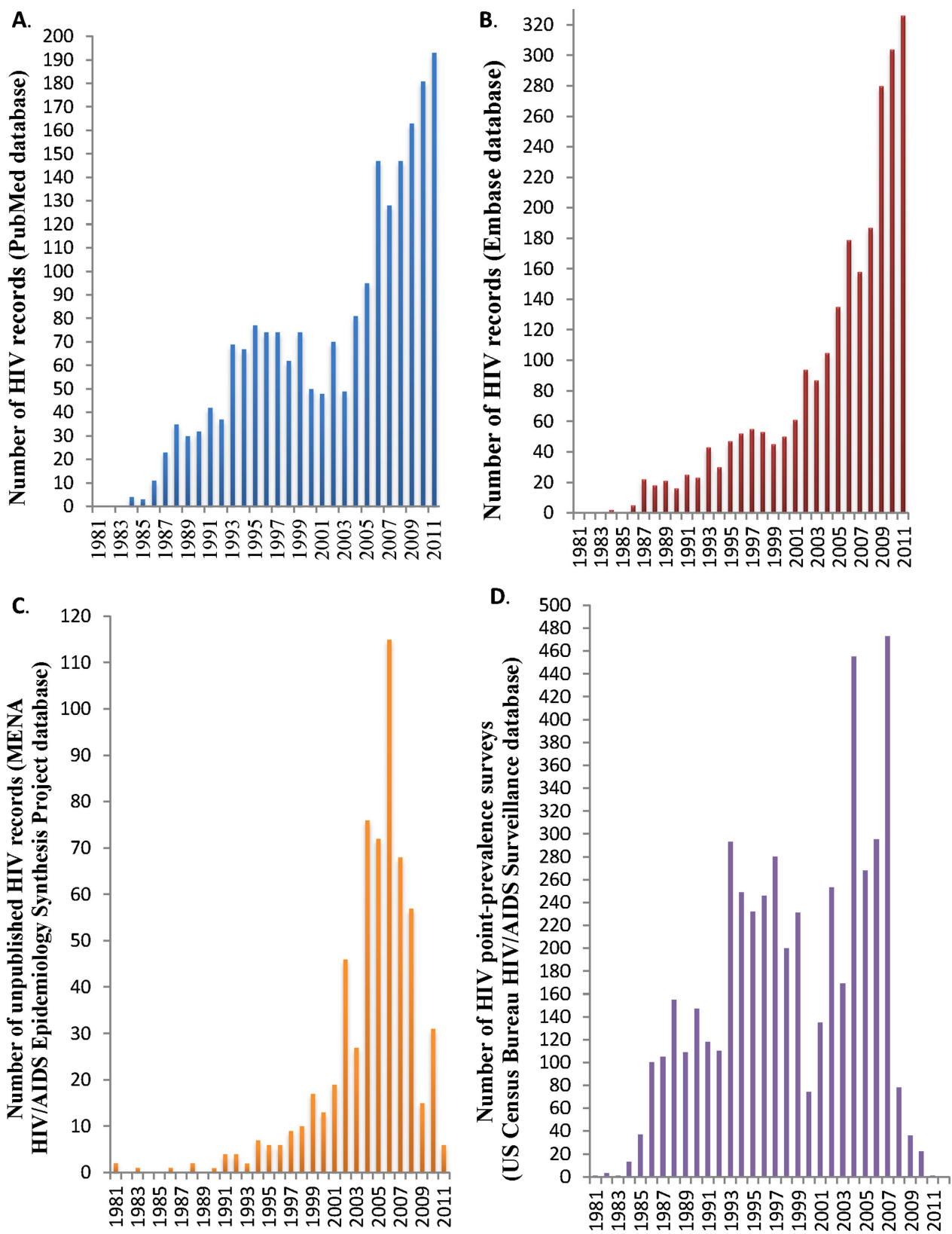

Figure 1 Progress of HIV research in the Middle East and North Africa (MENA) region since the discovery of the HIV epidemic. (A) Number of HIV records using the PubMed Index. (B) Number of HIV records using the Embase Index. (C) Number of HIV records using the MENA HIVIAIDS Epidemiology Synthesis Project Index. (D) Number of HIV point-prevalence surveys using the HIV Prevalence Index. Access the article online to view this figure in colour.

HIV is present and circulating in MENA countries was a taboo. ${ }^{4} 10^{11}$ It is not known, however, whether the ongoing global financial crisis, and the regional political instability, may impede this progress.

This progress, nevertheless, was not uniform across MENA countries. The last decade is marked by a widening disparity in HIV efforts (figure 2B). While few countries have made impressive strides, other countries continue to be far behind in terms of their will and capacity to conduct HIV research. Ironically, HIV research output does not appear to correlate with the country's per capita income. The Arabian Gulf countries that have the highest per capita income in the region have contributed minimally to this progress. Meanwhile, countries with constrained financial resources have made sizable contributions. In part, this reflects the fact that limited resource countries in the region, whenever they had strong commitment to advance HIV research, had access to funds through international donors such as the Global Fund to Fight AIDS, Tuberculosis and Malaria. ${ }^{12}$ This external support did not only provide Financial Resources to conduct studies, but most often contributed to capacity building within the countries through collaborative partnerships with international organisations working in the region, or through involvement of international consultants or experts. ${ }^{12}$

Morocco provides a notable example in this regards. Thanks to strong national commitment, and despite the limited resources, Morocco established surveillance systems with a national reach, submitted quality applications to the Global Fund that succeeded in securing financial and technical support and facilitated an environment for the civil society and non-governmental organisations to play an important role in HIV efforts. ${ }^{13} 14$

The Synthesis Project Index provides an indication of how a large volume of research in MENA never reaches the stage of 


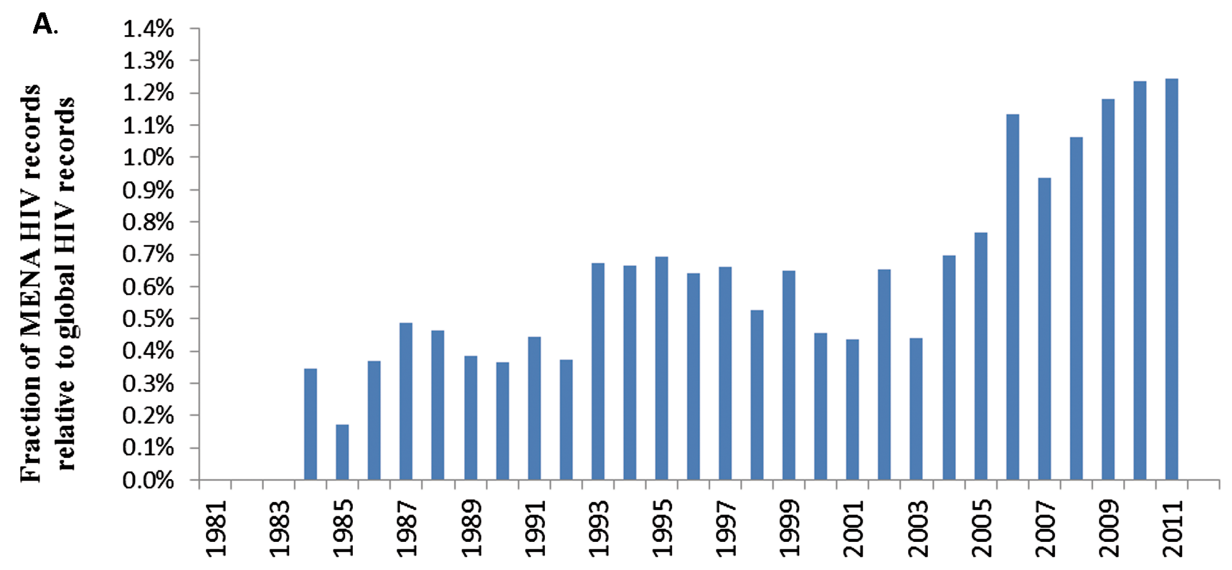

B.

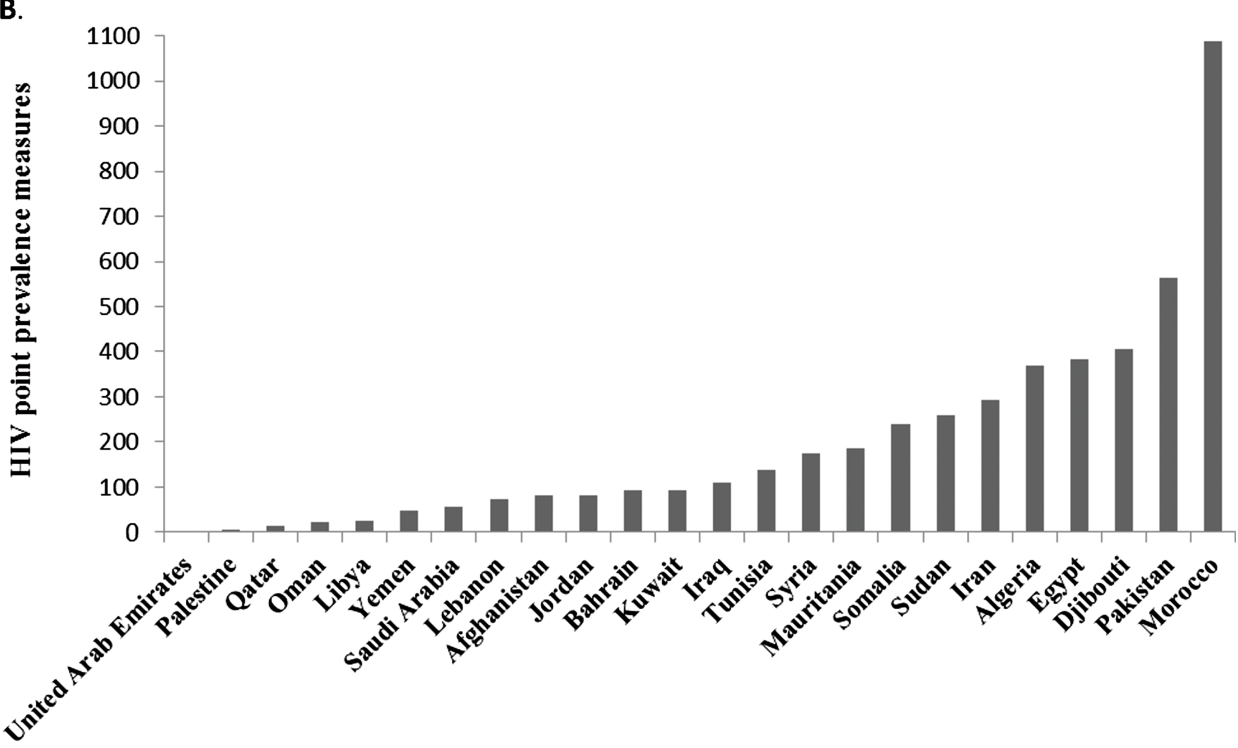

Figure 2 (A) Fraction of the Middle East and North Africa (MENA) HIV records in PubMed relative to global HIV records. (B) Distribution of HIV point-prevalence measures per country using the HIV Prevalence Index. Access the article online to view this figure in colour.

publication in scientific journals (figure 1). This is a structural weakness that reflects multiple factors, one of which is probably insufficient interest in academic publications. Many of the studies are conduced or supported through national programmes with limited interest in using the data beyond informing HIV policy and programmes at the national level. ${ }^{4} 6$ Limitations in the capacity to draft scientific publications, issues related to ownership of data and political sensitivity of the findings of some of the studies, may have further strained the efforts towards publication and dissemination to a wider audience.

Each of the four indices has its own limitations in capturing the diverse aspects that characterise the progress of HIV research in MENA. Some of the limitations in any specific index are addressed by the strengths of the other indices. However, this is not necessarily true for all limitations. For example, the total number of citations retrieved through PubMed and Embase is only a proxy of the number of publications that actually have primary data on HIV in the region. Nonetheless, screening of these two searches as part of previous systematic reviews conducted by the authors ${ }^{6-8} 13 \quad 1516$ confirmed a strong correlation between the total number of citations retrieved and the number of citations with actual data, with substantially more data being published in recent years.
Another limitation in our study is that the active systematic phase of the Synthesis Project to collect non-published HIV literature has ended in early 2009; a fact reflected in the drop in the number of records through this index after 2008. The drop accordingly does not reflect a true reduction in the number of new records of unpublished literature, as much as it is an artefact of the Synthesis Project process. Similarly, the drop in the HIV Prevalence Index after 2008 appears to reflect limitations in reporting to or updating the US Census Bureau HIV/ AIDS Surveillance database with new data entries in the last few years.

In conclusion, MENA has been witnessing a rapid growth in HIV research in the last few years despite significant challenges. Nevertheless, the region's contribution to global literature remains small relative to its population size and actual needs. There are knowledge gaps waiting to be filled, and the overall quality of studies still needs to be improved. Ongoing research efforts should be supported in a sustainable fashion to ensure the durability of the progress. Research should be conducted up to academic standard leading to scientific publications. Countries lagging in terms of research output should vigorously expand their research efforts to address the emerging HIV epidemics in this region. ${ }^{6} 7{ }^{15}$ Surveillance systems and research studies should be geared primarily towards high-risk populations 
who are most affected by the epidemic. Integrated biobehavioural surveillance surveys among these populations need to be conducted and repeated every few years. Risk-group size estimations, mapping and ethnographic studies and a better understanding of the sexual and injecting networks are warranted. General population sexual behaviour surveys will add useful data on the profile of risk behaviour in the population at large. Studies on HIV infected persons to improve case management are required. All of such studies are not only important for their direct benefits, but also important indirectly by facilitating and powering mathematical modelling and costeffectiveness analyses.

\section{Key messages}

- The Middle East and North Africa region has witnessed an impressive rapid growth in HIV research over the last decade.

- HIV research progress is heterogeneous across the countries of the region, and research output varies substantially from one country to another.

\section{Handling editor Jackie A Cassel}

Contributors HS managed the databases, collated the data, conducted the analyses and wrote the initial draft of the article. LJA conceived and led the design and conduct of the study. All authors contributed to the analysis, interpretation of the results, and writing of the article.

Funding The Qatar National Research Fund (NPRP 4-924-3-251) and the Biostatistics, Epidemiology, and Biomathematics Research Core at the Weill Cornell Medical College - Qatar.

\section{Competing interests None.}

Provenance and peer review Commissioned; externally peer reviewed.

Open Access This is an Open Access article distributed in accordance with the Creative Commons Attribution Non Commercial (CC BY-NC 3.0) license, which permits others to distribute, remix, adapt, build upon this work non-commercially, and license their derivative works on different terms, provided the original work is properly cited and the use is non-commercial. See: http://creativecommons.org/ licenses/by-nc/3.0/

\section{REFERENCES}

1 Bohannon J. Science in Libya. From pariah to science powerhouse? Science 2005;308:182-4

2 Kelley L, Eberstadt N. Behind the veil of a public health crisis: HIVIAIDS in the Muslim world. NBR Special Report 2005.

3 Kelley LM, Eberstadt N. The Muslim face of AIDS. Foreign Policy 2005;149:42-8.

4 Abu-Raddad L, Akala FA, Semini l, et al. Characterizing the HIVIAIDS epidemic in the Middle East and North Africa: Time for Strategic Action. Middle East and North Africa HIVIAIDS Epidemiology Synthesis Project. World Bank/UNAIDS/WHO Publication. Washington DC: The World Bank Press, 2010.

5 US Census Bureau International Database [database on the Internet]. Washington DC, USA. [cited May 2012]. http://www.census.gov/ipc/www/idb/ (accessed 9 Apr 2013).

6 Abu-Raddad LJ, Hilmi N, Mumtaz G, et al. Epidemiology of HIV infection in the Middle East and North Africa. AIDS 2010;24(Suppl 2):S5-23.

7 Mumtaz G, Hilmi N, McFarland W, et al. Are HIV epidemics among men who have sex with men emerging in the Middle East and North Africa?: a systematic review and data synthesis. PLoS Med 2011:8:e1000444.

8 Mumtaz G, Weiss $H$, Thomas $S$, et al. HIV epidemics are emerging among injecting drug users in the Middle-East and North Africa: systematic review and data synthesis. Under review. 2013.

9 Bozicevic I, Riedner G, Calleja JM. HIV surveillance in MENA: recent developments and results. Sex Transm Infect 2013:89:iii11-16.

10 Abu-Raddad L, Akala FA, Semini I, et al. Policy notes. Characterizing the HIVIAIDS epidemic in the Middle East and North Africa: Time for Strategic Action. Middle East and North Africa HIVIAIDS Epidemiology Synthesis Project. World Bank/ UNAIDS/WHO Publication. Washington DC: The World Bank Press, 2010.

11 Obermeyer CM. HIV in the Middle East. BMJ 2006;333:851-4.

12 Abu-Raddad LJ, Sgaier SK, Mumtaz RG. The HIV response in the Middle East and North Africa (MENA) region: an epidemic and its dilemmas. In: Smith RA, ed. Global HIVIAIDS politics, policy, and activism: persistent challenges and emerging issues. Santa Barbara, CA: Praeger, 2013. In press.

13 Kouyoumjian PS, Mumtaz RG, Hilmi N, et al. The epidemiology of HIV infection in Morocco: systematic review and data synthesis. Int J STD AIDS 2013. In press.

14 Mumtaz GR, Kouyoumjian SP, Hilmi N, et al. The distribution of new HIV infections by mode of exposure in Morocco. Sex Transm Infect 2013;89:iii49-56.

15 Mumtaz G, Hilmi N, Akala FA, et al. HIV-1 molecular epidemiology evidence and transmission patterns in the Middle East and North Africa. Sex Transm Infect 2011;87:101-6

16 Abu-Raddad LJ, Schiffer JT, Ashley R, et al. HSV-2 serology can be predictive of HIV epidemic potential and hidden sexual risk behavior in the Middle East and North Africa. Epidemics 2010;2:173-82. 